CERN-TH/99-136

\title{
THE HADRONIC TAU DECAY SIGNATURE OF A HEAVY CHARGED HIGGS BOSON AT LHC
}

\author{
D.P. ROY \\ Theoretical Physics Division, CERN \\ CH - 1211 Geneva 23 \\ and \\ Tata Institute of Fundamental Research, \\ Mumbai - 400005, India
}

\begin{abstract}
The hadronic tau decay channel offers by far the best signature for heavy charged Higgs boson search at the LHC in the large $\tan \beta$ region. By exploiting the distinct polarization of the tau and its large transverse mass, along with the accompanying missing- $p_{T}$, one can probe for a charged Higgs boson up to a mass of about $600 \mathrm{GeV}$ in an essentially background-free environment. The transverse mass distribution of the tau jet also provides a fairly unambiguous estimate of the charged Higgs boson mass.
\end{abstract}

CERN-TH/99-136

May 1999 
The charged Higgs boson carries the hallmark of a two Higgs doublet model and in particular the minimal supersymmetric Standard Model (MSSM). The two complex doublets correspond to eight scalar states, three of which are absorbed as Goldstone bosons. This leaves five physical particles - the two neutral scalars, a pseudoscalar and a pair of charged Higgs bosons $H^{ \pm}$[1]. While one of these neutral Higgs bosons may be hard to distinguish from that of the Standard Model, the $H^{ \pm}$carries a distinctive signature of the supersymmetric Higgs sector. Therefore the charged Higgs boson search is of considerable importance in probing the SUSY Higgs sector.

The charged Higgs boson couplings to fermions are given, in the diagonal Cabibbo-KobayashiMaskawa matrix approximation, by

$$
\mathcal{L}=\frac{g}{\sqrt{2} m_{W}} H^{+}\left\{\cot \beta m_{u_{i}} \bar{u}_{i} d_{i_{L}}+\tan \beta m_{d_{i}} \bar{u}_{i} d_{i R}+\tan \beta m_{\ell_{i}} \bar{\nu}_{i} \ell_{i R}\right\}+\text { H.C. },
$$

where $i$ denotes the generation index and $\tan \beta$ represents the ratio of the vacuum expectation values of the two Higgs doublets. The QCD corrections are taken into account, in the leading log approximation, by substituting the quark mass parameters by their running masses evaluated at the $H^{ \pm}$mass scale 2 .

It is clear from (11) that, for a charged Higgs boson lighter than the top quark,

$$
H^{+} \rightarrow \tau^{+} \nu
$$

is the dominant decay mode over the large $\tan \beta$ region. This is in striking constrast to the universal decay branching fractions of $W$ into each fermion pair. This difference has been utilized to search for $H^{ \pm}$in the top quark decay data of the CDF experiment in the $\tau$ channel at the Tevatron and obtain modest limits on the $H^{ \pm}$mass in the large $\tan \beta$ region [3]. The search can be extended up to $m_{H} \simeq m_{t}$ and over a wider region of $\tan \beta$ at the upgraded Tevatron and LHC, particularly by exploiting the opposite states of $\tau$ polarization coming from charged Higgs and $W$ boson decays [4, [5].

But the search for a heavy charged Higgs boson, $m_{H}>m_{t}$, at a hadron collider is generally considered very hard, because in this case the dominant decay mode

$$
H^{+} \rightarrow t \bar{b}
$$

suffers from a large QCD background. The dominant production process for a heavy $H^{ \pm}$at the LHC is its associated production with a top quark via gluon- $b$ quark fusion

$$
g \bar{b} \rightarrow H^{+} \bar{t}
$$

or the related process of gluon-gluon fusion

$$
g g \rightarrow H^{+} \bar{t} b
$$

Integrating out the kinematic variables of the final state $b$ quark in the second case results in a similar size of cross-section (within a factor of 2) as the first. This is as expected since $m_{b} \ll m_{t}, m_{H}$. The prospect of a heavy $H^{ \pm}$search at the LHC was considered in [6], [7] by 
combining the associated production of $H^{ \pm}$and $t$, with its dominant decay mode (3). The major background comes from the QCD processes of $t \bar{t} b$ or $t \bar{t} q(g)$, where the light quark or gluon jet could be misidentified as $b$. One could find a viable signature for the $H^{ \pm}$via triple $b$ tagging and reconstruction of $t, \bar{t}$ and $H^{ \pm}$masses; but only over a limited range of $\tan \beta$, i.e.,

$$
\tan \beta \sim 1 \quad \text { or } \quad \tan \beta \sim \frac{m_{t}}{m_{b}} .
$$

The reason why these two regions of $\tan \beta$ are favoured is the enhancement of the $t \bar{b} H^{ \pm}$coupling and the resulting signal cross-section as one can clearly see from (1)円. More recently the analysis has been extended to the $4 b$-tagged channel [9], by combining the associated production of $H^{+} \bar{t} b$ (5) with the dominant decay mode (3). Unfortunately, it requires the associated $b$ quark in (5) to be hard, which reduces the signal cross-section by a large factor. Consequently this signature is again viable only over the limited $\tan \beta$ regions of Eq. (6). It may be added here that the associated production of $H^{ \pm}$and $W^{\mp}$ bosons has been considered recently in [10], [11]. Being a second-order electroweak process, however, the size of the resulting signal is much smaller than (4), while it suffers from the same background. Consequently, it does not seem to offer a viable signal at the LHC [1].

The purpose of this work is to try to construct a viable signature for heavy $H^{ \pm}$search at the LHC via its $\tau$ decay mode (2). The importance of this decay mode for heavy $H^{ \pm}$search at the LHC has been recently emphasized in [12]. However, there is as yet no quantitative analysis of this signal along with the relevant background. For the signal we shall consider the associated production of $H^{ \pm}$with $t$ via $g b$ fusion followed by its $\tau$ decay, i.e.,

$$
g \bar{b} \rightarrow H^{+} \bar{t} ; \quad H^{+} \rightarrow \tau^{+} \nu, \quad \bar{t} \rightarrow \bar{b} q \bar{q}
$$

and its charge conjugate process. The major background comes from the $t \bar{t}$ production, i.e.,

$$
g g(q \bar{q}) \rightarrow t \bar{t}, \quad t \stackrel{W}{\rightarrow} b \tau^{+} \nu, \quad \bar{t} \rightarrow \bar{b} q \bar{q}
$$

and the charge conjugate final state. Even with a $50 \%$-tagging efficiency, the second $b$ in the background will escape identification most of the time; and this will be impossible to distinguish from a QCD jet accompanying the signal in the LHC environment. Thus we have to start with a signal/background ratio, which is even smaller than the $t \bar{t} b$ channel, considered in [6], [7].

As we shall see below, however, one can kinematically separate the signal from the background in this case by exploiting the hardness of the $\tau$-jet, its distinctive polarization and finally its large transverse mass with the accompanying missing- $p_{T}\left(\not p_{T}\right)$. It is to preserve this last feature that we have considered the hadronic decay of the accompanying top in (7) and (8).

The basic signal cross-section is obtained by multiplying the gluon- $b$ quark fusion crosssection with the hadronic BR of top decay $(=2 / 3)$ along with the $\mathrm{BR}$ of $H \rightarrow \tau \nu$, i.e.,

$$
B_{\tau}=\left[1+\frac{3 m_{b}^{2}}{m_{\tau}^{2}}\left(1+\frac{m_{t}^{2}}{m_{b}^{2} \tan ^{4} \beta}\right)\left(1-\frac{m_{t}^{2}}{m_{H}^{2}}\right)^{2}\right]^{-1}
$$

\footnotetext{
${ }^{1}$ Interestingly these two regions of $\tan \beta$ are favoured by GUT scale unification of $b$ and $\tau$ masses for a related reason; i.e., one needs a large Higgs Yukawa coupling in the RGE to control the growth of the $b$ mass at the low energy scale [8].
} 
As mentioned earlier, here $m_{b}$ stands for the running mass at the $H^{ \pm}$mass scale. It is adequate for our purpose to take 13

$$
m_{b}\left(m_{H}\right) \simeq m_{b}\left(m_{t}\right) \simeq 2.8 \mathrm{GeV} .
$$

The $m_{t}$ in the first factor of (9) comes from Yukawa coupling and in the second factor from phase space. The corresponding values should correspond to the running and the pole masses respectively, which differ by $5 \%$. For simplicity we shall put $m_{t}=175 \mathrm{GeV}$ for both and $m_{\tau}=$ $1.78 \mathrm{GeV}$. The resulting branching fraction is $B_{\tau}=0.7$ at $m_{H}=200 \mathrm{GeV}$, going down to 0.15 at $m_{H}=500 \mathrm{GeV}$, over the large $\tan \beta(\gtrsim 10)$ region of our interest.

We shall consider the hadronic decay channel of $\tau$, which carries a better imprint of its hardness and state of polarization, while the leptonic $(e, \mu)$ decay of $\tau$ from a $200-300 \mathrm{GeV} H^{ \pm}$ will look similar to the direct leptonic decay of a $W$ boson. We shall concentrate on the 1-prong hadronic decay of $\tau$, which is best suited to $\tau$ identification. It accounts for $80 \%$ of hadronic $\tau$ decay and $50 \%$ of its total decay width. The main contributors to 1-prong hadronic $\tau$ decay are

$$
\begin{aligned}
& \tau^{ \pm} \rightarrow \pi^{ \pm} \nu_{\tau}(12.5 \%), \\
& \tau^{ \pm} \rightarrow \rho^{ \pm} \nu_{\tau} \rightarrow \pi^{ \pm} \pi^{0} \nu_{\tau} \quad(26 \%), \\
& \tau^{ \pm} \rightarrow a_{1}^{ \pm} \nu_{\tau} \rightarrow \pi^{ \pm} \pi^{0} \nu_{\tau} \quad(7.5 \%),
\end{aligned}
$$

where the branching fractions for $\pi$ and $\rho$ channels include the small $K$ and $K^{*}$ contributions respectively [14, which have identical polarization effects. Together they account for a little over $90 \%$ of the 1-prong hadronic decay of $\tau$. The CM angular distribution of $\tau$ decay into $\pi$ or a vector meson $v\left(=\rho, a_{1}\right)$ is simply given in terms of its polarization as [4], [5]

$$
\begin{aligned}
& \frac{1}{\Gamma_{\pi}} \cdot \frac{d \Gamma_{\pi}}{d \cos \theta}=\frac{1}{2}\left(1+P_{\tau} \cos \theta\right), \\
& \frac{1}{\Gamma_{v}} \cdot \frac{d \Gamma_{v L}}{d \cos \theta}=\frac{\frac{1}{2} m_{\tau}^{2}}{m_{\tau}^{2}+2 m_{v}^{2}}\left(1+P_{\tau} \cos \theta\right), \\
& \frac{1}{\Gamma_{v}} \cdot \frac{d \Gamma_{v T}}{d \cos \theta}=\frac{m_{v}^{2}}{m_{\tau}^{2}+2 m_{v}^{2}}\left(1-P_{\tau} \cos \theta\right),
\end{aligned}
$$

where $P_{\tau}=+1(-1)$ for the $\tau$ coming from $H^{ \pm}\left(W^{ \pm}\right)$decay and $L, T$ denote the longitudinal and transverse polarization states of the vector meson. This angle is related to the fraction $x$ of $\tau$ lab. momentum carried by its decay meson, i.e., the (visible) momentum of the $\tau$-jet, via

$$
\cos \theta=\frac{2 x-1-m_{\pi, v}^{2} / m^{2} \tau}{1-m_{\pi, v}^{2} / m_{\tau}^{2}} .
$$

It is clear from Eqs. (14)-(17) that the effect of $\tau$ polarization is to give a harder $\tau$-jet from the signal relative to the background for the $\pi$ and the longitudinal vector meson contributions; but 
it is the other way round for the transverse part. The latter results in diluting the polarization effect by $50 \%$ for $\rho$ and practically washing it out for the $a_{1}$ contribution.

It is possible to suppress the transverse $\rho$ and $a_{1}$ contributions and enhance the difference between the signal and the background in the 1-prong hadronic $\tau$ decay channel even without identifying the individual mesonic contributions to this channel. The key feature of vector meson decays, relevant to this purpose, is that the transverse $\rho$ and $a_{1}$ favour even sharing of momentum among their decay pions, while the longitudinal $\rho$ and $a_{1}$ decays favour uneven distributions, where the charged pion carries either very little or most of the momentum. It is easy to derive this quantitatively for the $\rho$ decay. But one has to assume a dynamical model for the $a_{1}$ decay to get a quantitative result. We shall assume the model of Ref. [15], based on conserved axial vector current approximation, which provides a good descriptin of the $a_{1} \rightarrow 3 \pi$ data. A detailed account of the $\rho$ and $a_{1}$ decay formalisms can be found in [4], [5], along with the distributions of the resulting ratio,

$$
r=\frac{p_{\pi^{ \pm}}}{p_{\tau-j e t}}
$$

for the longitudinal and transverse $\rho$ and $a_{1}$. It suffices to state the main result here, i.e., this ratio is peaked near 0 and 1 for the longitudinal parts, but at the middle for the transverse parts. This suggests two ways of suppressing the transverse $\rho$ and $a_{1}$ contributions to the 1-prong hadronic $\tau$ decay channels [5]. The first one is to demand

$$
r=\frac{p_{\pi^{ \pm}}}{p_{\tau-j e t}}>0.8
$$

i.e., over $80 \%$ of the $\tau$-jet $p_{T}$ is carried by the charged track. It retains only the $\pi$ and half of $\rho_{L}$ contribution, while sacrificing the other half of $\rho_{L}$ and $a_{1 L}$ along with the $\rho_{T}$ and $a_{1 T}$. But it gives a robust signature of a very hard single track, accounting for over $80 \%$ of the calorimetric energy deposit. The second method is to demand a hard distribution in

$$
\Delta p_{T}=\left|p_{T}^{\pi^{ \pm}}-p_{T}^{\pi^{0}}\right|
$$

i.e., the difference of momenta carried by the charged track and the accompanying neutral pion(s) instead of their sum. It retains most of the $\rho_{L}$ and $a_{1 L}$ contributions along with the $\pi$, since it includes both $r \simeq 1$ and $r \simeq 0$ regions. However, the latter corresponds to a soft charged track along the direction of a hard calorimetric energy deposit, which could be deflected away from this direction by the magnetic field. Note that this part is present even in the normal $p_{T}$ distribution of the 1-prong $\tau$-jet, but not the one satisfying (19). Hopefully this problem will be taken care of in a more sophisticated analysis including detector simulation. For the present purpose we shall illustrate the enhancement of signal/background ratio via both methods, without worrying about this problem.

Our results are based on a parton level Monte Carlo calculation of the signal and background processes (7) and (8), followed by the 1-prong hadronic decay of $\tau$ via $\pi, \rho$ and $a_{1}$. The LO cross-sections for the signal and background processes are convoluted with the LO parton distributions of CTEQ 4L [16] using the threshold energies, $\left(m_{H}+m_{t}\right)$ and $2 m_{t}$, as the scale 
Table 1: Charged Higgs signal and background cross-sections in the 1-prong hadronic $\tau$ plus multijet channel for $\tan \beta=40$. Here $p_{T}$ refers to the visible $p_{T}$ of the $\tau$, while $p_{T}^{c}$ and $\Delta p_{T}$ are defined in the text. The last row shows the $\tau$ branching fractions of the signal and the background, which are included in these cross-sections.

\begin{tabular}{|l|c|c|c|c|}
\hline$\sigma(\mathrm{fb})$ & $H^{+}(200 \mathrm{GeV})$ & $H^{ \pm}(400 \mathrm{GeV})$ & $H^{ \pm}(600 \mathrm{GeV})$ & $B g$ \\
\hline $\begin{array}{l}\text { Basic Cuts } \\
(\text { Eqs. 21-23) }\end{array}$ & 580 & 30.1 & 6.7 & 16176 \\
\hline$p_{T}>100 \mathrm{GeV}$ & 132 & 17.7 & 5.1 & 720 \\
\hline$p_{T}^{c}>100 \mathrm{GeV}$ & 61.1 & 8.1 & 2.3 & 124 \\
\hline$\Delta p_{T}>100 \mathrm{GeV}$ & 68.6 & 10.7 & 3.6 & 136 \\
\hline$B_{\tau}$ & 0.71 & 0.17 & 0.14 & $2 \times 0.11$ \\
\hline
\end{tabular}

parameter. We have checked that the resulting cross-sections are 10-20\% higher than those obtained with the NLL parton distributions of $\operatorname{MRS}(\mathrm{G})$ [17]. The parton distributions were used via the CERN pdflib version 7.09.

We have tried to simulate detector resolution by a gaussian smearing of $p_{T}$, with $\left(\sigma\left(p_{T}\right) / p_{T}\right)^{2}=$ $\left(0.6 / \sqrt{p_{T}}\right)^{2}+(0.04)^{2}$, for all the jets including the $\tau$-jet. The $p_{T}$ is obtained by vector addition of all these $p_{T}$ 's after the gaussian smearing. As a basic set of selection cuts we require

$$
p_{T}>20 \mathrm{GeV} \text { and } \eta<3
$$

for all the jets, where $\eta$ denotes pseudorapidity. Only the $b$-jet accompanying $\tau$ in the background process (8) is not subject to this constraint. We also require a minimum separation of

$$
\Delta R=\left[(\Delta \phi)^{2}+(\Delta \eta)^{2}\right]^{1 / 2}>0.4
$$

between each pair of jets, where $\phi$ denotes the azimuthal angle. The jets are required to satisfy the $W$ and top mass constraints, i.e.,

$$
\left|m_{j j}-m_{W}\right|<15 \mathrm{GeV}, \quad\left|m_{j j j}-m_{t}\right|<25 \mathrm{GeV} .
$$

With the above resolution smearing, these mass constraints are satisfied essentially without any loss to the signal or background. We shall also require the third jet, entering the top mass constraint, to be $b$-tagged for an unambiguous identification of the accompanying top. The signal and background cross-sections presented below are to be multiplied by the corresponding efficiency factor, which may be optimistically assumed to be $\sim 0.5$.

Table 1 shows the signal cross-sections for $m_{H}=200,400$ and $600 \mathrm{GeV}$ at $\tan \beta=40$ along with the $\bar{t} t$ background. The cross-sections, obtained with the basic selection cuts of Eqs. (21)(23), are shown in the first row. At this stage the background is 2 to 3 orders of magnitude larger than the signal. The second row shows the cross-sections after a

$$
p_{T}>100 \mathrm{GeV}
$$


cut on the $\tau$-jet. As expected, it reduces the background more severely than the signal, so that it is now 1 to 2 orders of magnitude larger than the signal. The signal/background ratio can be improved further by exploiting the $\tau$ polarization effect as discussed above. The third row shows the cross-section for

$$
p_{T}^{c}>100 \mathrm{GeV},
$$

where $p_{T}^{c}$ denotes the $p_{T}$ of the $\tau$-jet satisfying (19), i.e., where the charged track carries over $80 \%$ of the $p_{T}$. It reduces the background by a factor of 6 , while costing a factor of 2 to the signal. The fourth row shows the cross-sections for

$$
\Delta p_{T}>100 \mathrm{GeV}
$$

The signal and background cross-sections in this case are similar to the previous case.

Figures 1a,b,c show the distributions of the signal and background cross-sections against $p_{T}, p_{T}^{c}$ and $\Delta p_{T}$ respectively. As expected, the signal cross-sections get harder with increasing $H^{ \pm}$mass. One can also see the hardness of the signal cross-section relative to the background increasing as one goes from $p_{T}$ to $p_{T}^{c}$ or $\Delta p_{T}$. Thus the $p_{T}$ distribution of the background is seen to remain higher than the signal over the entire range; but the $p_{T}^{c}$ distribution of the $200 \mathrm{GeV}$ $H^{ \pm}$signal overtakes the background, and even the 400 and $600 \mathrm{GeV} H^{ \pm}$signals catch up with the latter. The same is true for the $\Delta p_{T}$ distributions. However, the distribution in $p_{T}^{c}$ may be of greater significance in view of the robustness of this variable.

Figure 2 shows the signal and background cross-sections, with $p_{T}^{c}>100 \mathrm{GeV}(25)$, against the azimuthal angle of the $\not p_{T}$ relative to the $\tau$-jet. The background ( $W \rightarrow \tau \nu$ ) events, satisfying the large $p_{T}^{c}$ cut, necessarily require a large $p_{T}$ boost for the $W$ boson. This results in a small opening angle between its decay products. In contrast the $H^{ \pm} \rightarrow \tau \nu$ events require only a modest $p_{T}$ boost for $M_{H}=200 \mathrm{GeV}$ and none for the larger $H^{ \pm}$masses. This accounts for the flat azimuthal opening angle distribution between the $\tau$ and $\not p_{T}$ for the $200 \mathrm{GeV} H^{ \pm}$signal and backward peaks for the higher masses. One gets similar results for the signal and background cross-sections with $p_{T}$ or $\Delta p_{T}$ cuts of $100 \mathrm{GeV}$.

Similarly the $\not p_{T}$ distribution of the signal is harder than the background; and the difference increases with increasing $H^{ \pm}$mass. The quantity in which the cumulative difference between the signal and the background becomes most striking is the transverse mass of the $\tau$-jet with the $\not p_{T}$, i.e.,

$$
m_{T}=2 p_{T} p_{T}(1-\cos \phi), \quad m_{T}^{c}=2 p_{T}^{c} p_{T}(1-\cos \phi),
$$

since it is kinematically constrained to be smaller than the parent $H^{ \pm}(W)$ mass. The $m_{T}$ and $m_{T}^{c}$ distributions of the signal and background cross-sections are shown in Fig. 3a,b, which include the $p_{T}\left(p_{T}^{c}\right)>100 \mathrm{GeV}$ cut. There is a clear separation between the signal and the background cross-sections, particularly in Fig. 3b. It thus seems possible to practically eliminate the background via a suitable $m_{T}^{c}$ cut with very little cost to the signal cross-section. Consequently one can probe for the $H^{ \pm}$signal in an essentially background-free environment. It is also evident from Fig. 3a,b that the transverse mass distribution can provide a fairly unambiguous estimate of the $H^{ \pm}$mass. In comparing Fig. 3a with 3b, one sees a significantly larger background in the former case, resulting in a larger encroachment into the signal region. 
Thus the separation of the signal and background in the $m_{t}$ distribution may be less clean, particularly if the uncertainty in the $p_{T}$ turns out to be larger than that suggested by the assumed resolution smearing. On the other hand, if it is possible to separate the two from the $m_{T}$ distribution, then the polarization effect can be used as a confirmatory test of the signal. We have checked that the background cross-section at large $m_{T}(>100 \mathrm{GeV})$ is dominated by the transverse $\rho$ and $a_{1}$ contributions. Consequently its distribution in the ratio $r$ (18) vanishes at $r \simeq 0$ and 1 while peaking in the middle. In contrast the signal is dominated by the $\pi, \rho_{L}$ and $a_{1 L}$ contributions, showing peaks at $r \simeq 0$ and 1 , and a dip in the middle.

Assuming that the signal can be clearly separated from the background from the $m_{T}$ or $m_{T}^{c}$ distribution, the $H^{ \pm}$discovery limit of the LHC will be primarily determined by the signal size. The size of the signal cross-sections shown in Fig. 3a,b corresponds to those listed in the second and third rows of Table 1 . They have to be multiplied by the $b$-tagging efficiency of $\sim 0.5$. Still one expects a signal size of at least $1 \mathrm{fb}$ up to $m_{H}=600 \mathrm{GeV}$. This will correspond to at least 100 events with the expected annual luminosity of $100 \mathrm{fb}^{-1}$ for the high luminosity run of the LHC - i.e., a total of at least 300-400 events over a period of 3-4 years. Of course we have concentrated so far on a favourable value of $\tan \beta=40$. For lower values of $\tan \beta$ the signal cross-section is expected to go down like $\tan ^{2} \beta$. Thus one expects a total of at least 100 (25) signal events at $\tan \beta=20(10)$, for a $H^{ \pm}$mass going up to $600 \mathrm{GeV}$. Hence the hadronic $\tau$ decay channel seems to offer a viable signature for the $H^{ \pm}$search at the LHC up to $600 \mathrm{GeV}$ over a sizeable part of the large $\tan \beta$ region, i.e., $\tan \beta \gtrsim 10$.

In summary, we have analysed the prospect of a heavy $H^{ \pm}$search at the LHC via its hadronic $\tau$ decay channel. The dominant signal comes from the associated production of $H^{ \pm}$with a top quark, while the dominant background is from top pair production. Although the signal is 2 to 3 orders of magnitude smaller than the background, it can be effectively separated by exploiting the hardness and the distinctive polarization of the $\tau$ coming from $H^{ \pm}$decay. In particular an effective method of utilizing the polarization effect is to select the 1-prong hadronic $\tau$ jets, where the charged prong carries over $80 \%$ of its visible momentum. The resulting transverse mass of the $\tau$ jet with the accompanying $p_{T}$ shows a clear separation between the signal and the background, as well as providing a fairly unambiguous estimate of the $H^{ \pm}$mass. Thus the viability of the signal is primarily determined by the signal size. For the high luminosity run of the LHC, one expects a viable signal up to the $H^{ \pm}$mass of $600 \mathrm{GeV}$ over the large $\tan \beta(\gtrsim 10)$ region.

I thank Daniel Denegri for persuading me to undertake this investigation. I also thank Michelangelo Mangano, Debajyoti Choudhury and Sunanda Banerjee for discussions and Swagata Banerjee for help with the figure-plotting programme. This work was partly supported by the IFCPAR under project No. 1701-1 on Collider Physics. 


\section{References}

[1] J.F. Gunion, H.E. Haber, G.L. Kane and S. Dawson, "The Higgs Hunters' Guide" (Addison-Wesley, Reading, MA, 1990).

[2] M. Drees and D.P. Roy, Phys.Lett. B269 (1991) 155.

[3] M. Guchait and D.P. Roy, Phys.Rev. D55 (1997) 7263;

CDF Collaboration: F. Abe et al, Phys.Rev.Lett. 79 (1997) 357.

[4] B.K. Bullock, H. Hgiwara and A.D. Martin, Nucl.Phys. B395 (1993) 499.

[5] S. Raychaudhuri and D.P. Roy, Phys.Rev. D52 (1995) 1556; D53 (1996) 4902.

[6] V. Barger, R.J.N. Phillips and D.P. Roy, Phys.Lett. B324 (1994) 236.

[7] J.F. Gunion, Phys.Lett. B322 (1994) 125.

[8] See e.g., S. Dimopoulos, L.J. Hall and S. Raby, Phys.Rev. D45 (1992) 4192;

V. Barger, M.S. Berger and P. Ohman, Phys.Rev. D47 (1993) 1093.

[9] D.J. Miller, S. Moretti, D.P. Roy and W.J. Stirling, "Detecting heavy charged Higgs bosons at the LHC with four b-tags", in preparation.

[10] A.A. Barrientos Bendezu and B.A. Kniehl, Phys.Rev. D59 (1999) 015009.

[11] S. Moretti and K. Odagiri, Phys.Rev. D59 (1999) 055008.

[12] K. Odagiri, hep-ph/9901432.

[13] H. Fusaoka and Y. Koide, Phys.Rev. D57 (1998) 3986.

[14] Review of Particle Properties, C. Caso et al., Eur.Phys.J. C3 (1998) 1.

[15] J.H. Kühn and A. Santamaria, Z.Phys. C48 (1990) 445.

[16] CTEQ Collaboration: H.L. Lai et al., Phys.Rev. D55 (1997) 1280.

[17] A.D. Martin, R.G. Roberts and W.J. Stirling, Preprint RAL, RAL-95-021 (1995). 

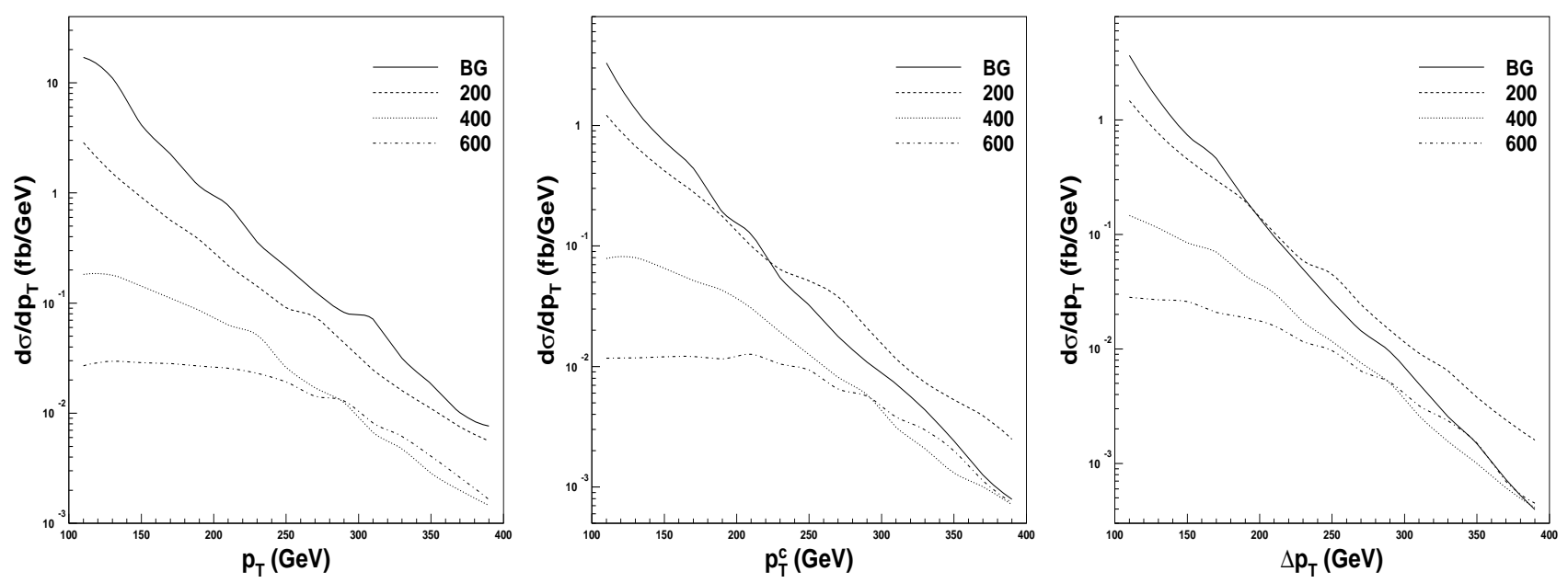

Figure 1: Distributions of the $H^{ \pm}$signal and the background cross-sections in a) $p_{T}$ of the $\tau$-jet, b) $p_{T}$ of those $\tau$-jets where the charged pion carries over $80 \%$ of the $p_{T}$, c) difference of the charged and neutral pion contributions to the $p_{T}$ of the $\tau$-jet. The signal cross-sections in this and the following figures correspond to $\tan \beta=40$. 


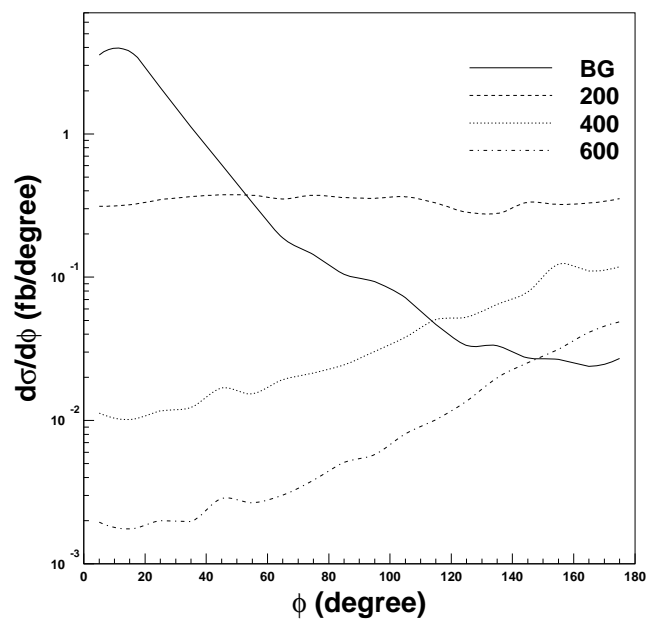

Figure 2: Distribution of the $H^{ \pm}$signal and background cross-sections, satisfying $p_{T}^{c}>100 \mathrm{GeV}$, in the azimuthal angle between the $\tau$-jet and the missing- $p_{T}$.
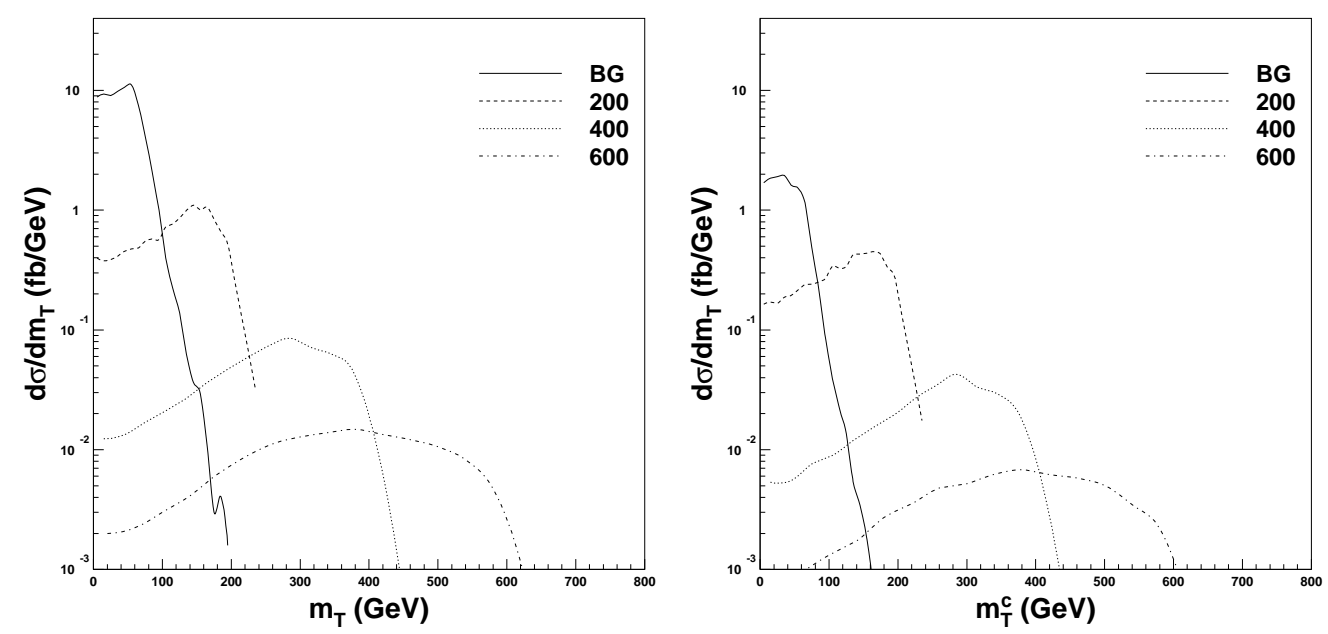

Figure 3: Distribution of the $H^{ \pm}$signal and background cross-sections in the transverse mass of the $\tau$-jet with the missing- $p_{T}$ for a) all 1-prong $\tau$-jets, b) those where the charged pion carries over $80 \%$ of the $\tau$-jet $p_{T}$. 